\title{
A MICROMECHANICAL MODEL OF TENSION- SOFTENING AND BRIDGING TOUGHENING OF SHORT RANDOM FIBER REINFORCED BRITTLE MATRIX COMPOSITES
}

\author{
Victor C. Li, $\dagger$ Youjiang Wang $\ddagger$ and Stanley BaCker $\$$ \\ $\dagger$ Advanced Civil Engineering Materials Research Laboratory, Department of Civil Engineering, The \\ University of Michigan, Ann Arbor, MI 48109-2125, U.S.A.; † School of Textile and Fiber Engineering, \\ Georgia Institute of Technology, Atlanta, GA 30332-0295, U.S.A. and § Department of Mechanical \\ Engineering, Massachusetts Institute of Technology, Cambridge, MA 02139, U.S.A.
}

(Received 3 October 1989 ; in revised form 19 April 1990)

\begin{abstract}
A MICROMECHANICAL model has been formulated for the post-cracking behavior of a brittle matrix composite reinforced with randomly distributed short fibers. This model incorporates the mechanics of pull-out of fibers which are inclined at an angle to the matrix crack plane and which undergo slip-weakening or slip-hardening during the pull-out process. In addition, the random location and orientation of fibers are accounted for. Comparisons of model predictions of post-cracking tension-softening behavior with experimental data appear to support the validity of the model. The model is used to examinte the effects of fiber length, snubbing friction coefficient and interfacial bond behavior on composite post-cracking tensile properties. The scaling of the bridging fracture toughening with material parameters is discussed.
\end{abstract}

\section{NOMENCLATURE}

$A_{\mathrm{f}} \quad$ fiber cross-sectional area

$d_{f} \quad$ fiber diameter

$E_{\mathrm{f}} \quad$ fiber elastic modulus

$f$ snubbing friction coefficient

$G \quad$ composile fracture energy

$\ell \quad$ short embedded length of fiber

$L_{\mathrm{c}} \quad$ critical fiber embedded length of pull-out

$L_{\mathrm{f}} \quad$ fiber length

$P \quad$ force in fiber segment bridging across matrix crack

$s \quad$ local slippage on fiber-matrix interface

$V_{\mathrm{f}} \quad$ fiber volume fraction

$z \quad$ distance of centroid of fibcr from crack plane

$\delta \quad$ matrix crack opening

$\phi \quad$ orientation of fiber with respect to tensile loading direction

$\sigma_{f}^{\mu} \quad$ fiber strength

$\tau \quad$ bond strength of fiber-matrix interface, can be made a function of $s$ 


\section{INTRODUCTION}

IN QUASI-BRITTLE materials such as concrete and rock, and certain brittle matrix composites reinforced with short fibers or whiskers, the tensile behavior after peak strength may be represented by a tension-softening curve. This curve describes the decreasing traction versus crack-opening relationship and the area under it has been related to the material critical fracture energy release rate (RICE, 1968). Development of the fracture process zone and hence the $R$-curve behavior have been shown to be controlled by the tension-softening curve (LI and LianG, 1986). Further, the tensionsoftening curve has been related to structural properties such as modulus of rupture (MOR) values and shear strength of beams (HILLERBORG, 1989; W ARD and LI, 1990; LI and WARD, 1990).

In recent experimental studies, WANG ef $a$. (1990a, b) found that the tensionsoftening curve of cement mortar can be significantly enhanced by the inclusion of a small volume percentage of synthetic fibers. Different fiber types (aramids, polyethylene, polypropylene) induce different failure mechanisms (WANG et al.. 1990c), leading to different shapes of the tension-softening curve. In composites with good fiber dispersion, the failure mechanism is controlled by fiber pull-out or rupture, depending on fiber geometry and interfacial bond strength. Such behavior is exhibited by low volume fraction reinforcement $(<2 \%)$ of Spectra 900 (an ultrastrength polyethylene fiber), polypropylene fibers, and steel fibers.

There are two major motivations for the development of constitutive models relating the microstructural parameters to the mechanical behavior of fiber composites. One is to guide the optimization of material behavior by tailoring the types and forms of the constituent components, and the other is to predict the mechanical response of end products made of such materials. While pre-peak stress-strain behavior and associated mechanical properties of fiber composites (particularly those with continuous aligned fiber reinforcement) have been extensively studied (e.g. AvESTON 't al., 1971; Budiansky et al., 1986; Gopalaratnam and SHAH, 1987), the post-peak tension-softening behavior of fiber-reinforced composites has not been adequately investigated. However, the work of fracture due to bridging of discontinuous but aligned fibers in brittle matrix composites was studied by CoTtrell (1964), COOPER and KeLLy (1970) and KFLLY and MACMILLAN (1986), and that of discontinuous and random fibers was studied by WETHERHOLD (1989). In this paper, a statistical-micromechanical model of the tension-softening behavior of short fiber-reinforced composites is constructed based on the random nature of fiber distribution and accounting for dominant features of the composite failure mechanisms. These features include fibers pulled out at an angle to the matrix crack plane as well as slip-weakening or hardening of the fiber interface during the pull-out process. The composite bridging toughness may be obtained from the area under the tension-softening curve. In the following the model assumptions are given first. Then development of the model is presented. Certain characteristics of predicted tension-softening curves and fracture energy are examined in the light of fiber length optimization. Finally, model-predicted tension-softening curves as a function of microstructural parameters are compared with experimental data from Spectra fiber-reinforced mortar. 


\section{Problem Formulation}

\section{Model assumptions}

The following assumptions are adopted: (1) the matrix crack is planar, (2) the matrix deformation is negligible during fiber pull-out, (3) the fibers have 3-D random distribution in location and orientation, (4) the fibers are straight with cylindrical geometry, (5) the fibers behave linear elastically, (6) the fibers rupture when their axial stress reaches the fiber strength $\sigma_{f}^{u}$, (7) the Poisson effect of the fiber on pull-out is neglected, (8) the fiber-matrix bond is frictional and the elastic bond strength is neglected. The frictional bond strength may exhibit slip-hardening or weakening behavior. In addition, the model assumes that the effect of fiber pull-out from matrix at an oblique angle can be characterized by a snubbing friction coefficient, $f$. These last two assumptions are based on recent experimental observations. Since this model only predicts the tensile stress vs crack separation $(\sigma-\delta)$ curve, no assumptions regarding matrix bulk properties are made.

It should be noted that some of the above assumptions hold true only for certain fiber types, while others, at best, approximate the real behavior. For example, the model should not be used for fibers which undergo extensive yielding prior to fiber rupture, such as undrawn polypropylene fibers. Also, for some composites, the presence of fibers lying at a high angle to the tensile loading axis may lead to crack plane deflection parallel to the fiber-matrix interface. These aspects are not accounted for in the present work. In addition, when the fiber stiffness is high and when large fiber volume fraction is used, the elasticity of the matrix material must be taken into account, even in the post-cracking stage, such as in shear lag models (see e.g. GopALARATNAm and SHah, 1987; GaO et al., 1988). However, as will become clear later, the modular nature of the model development does allow the flexibility of relaxing some of these assumptions if necessary.

\section{Bridging fibers}

Figure 1 shows a fiber of length $L_{\mathrm{f}}$ arbitrarily located with its centroid at a distance $z$ from the matrix crack plane, and with an orientation angle $\phi$ to the tensile loading axis. Only fibers with a positive embedded length $\ell$, defined by

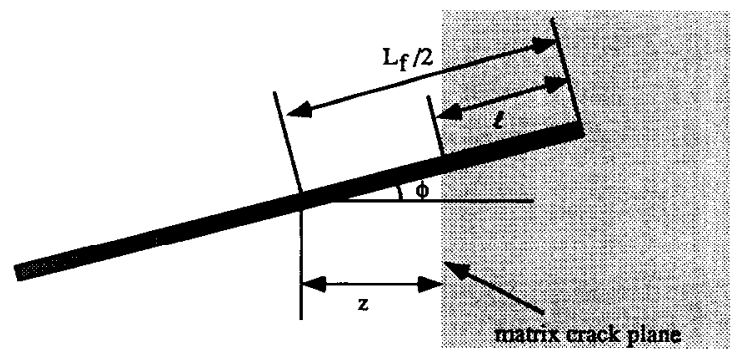

FIG. 1. A fiber crossing a matrix crack. 

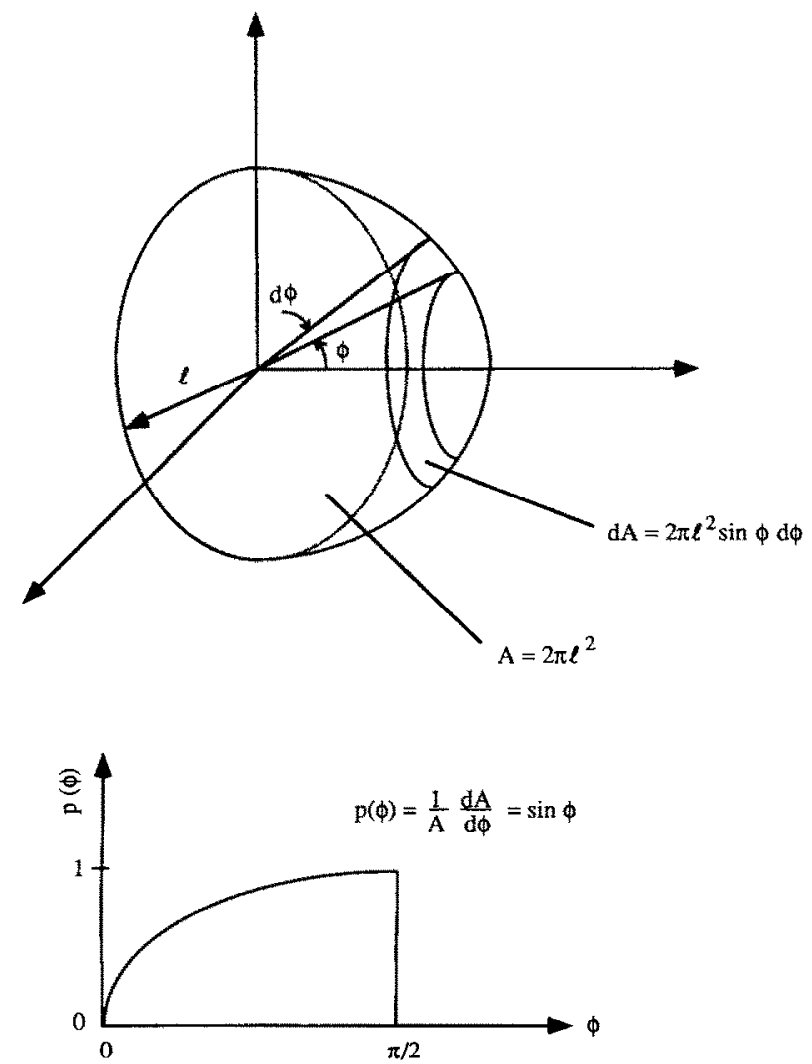

Fig. 2. 3-D randomly orientated fibers with embedded length $t$ have their fiber ends lying on the hemispherical surface. Also shown is the corresponding probability density function $p(\phi)$.

$$
\ell=\frac{L_{\mathrm{f}}}{2}-\frac{z}{\cos \phi}
$$

would cross the crack plane and provide the bridging action. This implies the crack interception condition

$$
\phi \leqslant \arccos \frac{2 z}{L_{\mathrm{f}}} .
$$

The number of fibers bridging across a given matrix crack plane clearly depends on the distribution of $z$ and $\phi$. For a uniform random distribution, the probability density function $p(z)$ of the centroidal distance $z$ is simply

$$
p(z)=\frac{2}{L_{\mathrm{f}}} \text { for } 0 \leqslant z \leqslant L_{\mathrm{f}} / 2 .
$$

The statement of three-dimensional random orientation of fibers in the matrix is equivalent to saying that a fiber end has an equal likelihood of being located at any point on a hemisphere, as shown in Fig. 2. According to that figure, the probability density function, $p(\phi)$, of the inclining angle $\phi$ is given by 


$$
p(\phi)=\sin \phi \text { for } 0 \leqslant \phi \leqslant \pi / 2 .
$$

Thus, the number of fibers $\mathrm{d} N$ with orientation angle $\phi$ to $\phi+\mathrm{d} \phi$ and located at $z$ to $z+\mathrm{d} z$ bridging across the crack may be calculated from

$$
\mathrm{d} N=N_{\mathrm{t}} p(\phi) \mathrm{d} \phi p(z) \mathrm{d} z \text { for } 0 \leqslant \phi \leqslant \arccos \left(2 z / L_{\mathrm{f}}\right) \text { and } 0 \leqslant z \leqslant L_{\mathrm{f}} / 2 \text {, }
$$

where $N_{\mathrm{t}}$ is the total number of fibers in the matrix of volume $A_{\mathrm{c}} L_{\mathrm{f}}$ which contains fibers bridging the matrix crack plane of area $A_{\mathrm{c}}$. Thus,

$$
N_{\mathrm{t}}=\frac{\text { total fiber volume }}{\text { volume per fiber }}=\frac{A_{\mathrm{c}} L_{\mathrm{f}} V_{\mathrm{f}}}{L_{\mathrm{f}} A_{\mathrm{f}}}=\frac{A_{\mathrm{c}}}{A_{\mathrm{f}}} V_{\mathrm{f}},
$$

where $A_{\mathrm{f}}$ is the fiber cross-sectional area and $V_{\mathrm{f}}$ is the fiber volume fraction in the composite.

Equation (5) already implicitly assumed that the fiber location $z$ and the fiber angle $\phi$ are independent variables. In order to calculate the total force transmission across the matrix crack, it would be necessary to analyse the bridging force $P$ carried by a single fiber with an embedded length $\ell$ and fiber angle $\phi$. It should be noted that the value of $\ell$ is implied once $z$ and $\phi$ are specified (see Eq. 1). Clearly, the fiber force first increases as the interface-debonded zone extends towards the embedded end, and then decreases as the fiber slips out in association with an increasing matrix crack width $\delta$. Hence, for given fiber geometry and property, $P=P(\ell, \phi, \delta)$.

\section{Bridging forces $\mathrm{P}(\ell, \phi, \delta)$}

The problem of fiber pull-out at $\phi=0$ is illustrated in Fig. 3(a). To include the possibility of fiber abrasion (WANG et al., 1988a) or interface smoothing (as may occur in strong fiber-weak matrix composite systems) we shall adopt the Wang-LiBacker model of two-sided fiber pull-out (WANG et al., 1988b). This model assumes a frictional bond at the fiber-matrix interface, neglecting any effect of an elastic bond. However, the frictional resistance is made to depend on the amount of slip so that either interface slip-weakening or hardening phenomenon can be incorporated.

The solution procedure is illustrated in Fig. 4 for the case of slip-hardening frictional bond. In this case, the short embedded end will be completely pulled out, whereas the long embedded end may have had some slippage. Thus, the total crack opening $\delta=\delta_{1}+\delta_{2}$ at the end of the loading process may exceed the length of the short embedded end $\ell_{1}$.

The $\delta_{1}$ and $\delta_{2}$ may be regarded as load point displacement of loads $P_{1}$ and $P_{2}$ directly applied to the ends of the two embedded lengths of fiber. In reality, the two fiber segments are connected and equilibrium enforces the condition that $P_{1}$ must always equal $P_{2}$. This implies that, once the short fiber segment load $P_{1}$ reaches a maximum $P_{\max }$, the long fiber segment must also unload with partial retrieval of the fiber segment bridging the matrix crack back into the matrix, rather than following the complete direct pull-out curve indicated by the dashed line in Fig. 4 .

The result of this analysis is a $P-\delta$ relationship which depends on fiber length $L_{\mathrm{f}}$, diameter $d_{\mathrm{f}}$ and elastic mudulus $E_{\mathrm{f}}$, as well as on the embedded length $\ell_{1}$ or $\ell_{2}$, and the slip-dependent frictional resistance $\tau(s)$. WANG et al. (1988b) found that the model 


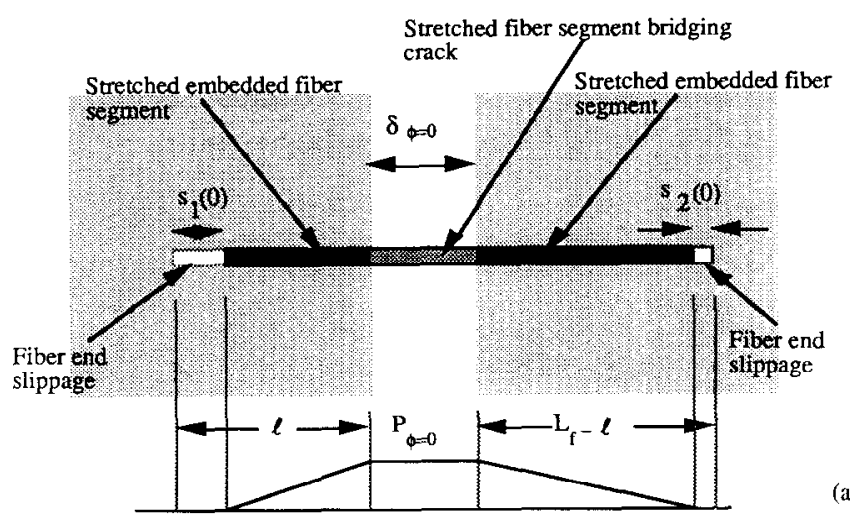

(a)

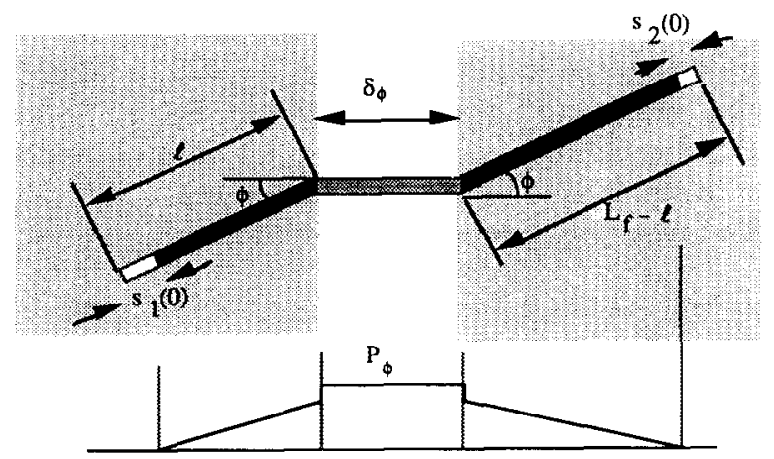

(b)

Fig. 3. Two-sided pull out for a fiber with length $L_{\mathrm{f}}$ and short embedded length $\ell$, and with end slippages $s_{1}(0)$ and $s_{2}(0)$, for fibers (a) aligned in the direction of the tensile loading axis and (b) oriented at an arbitrary angle $\phi$ to the loading axis. In both cases, the axial force along the fiber length is shown.

requires a slip-hardening $\tau-s$ relationship in order to match data of certain synthetic fibers, while a slip-weakening $\tau-s$ relationship is required to match data from steel fibers. Figure $5(\mathrm{a}, \mathrm{b})$ shows the experimentally determined $P-\delta$ curves for a synthetic (nylon) fiber and for a steel fiber pulled out from a cement matrix, and the corresponding model $\tau-s$ and predicted $P-\delta$ curves. The stecl fibcr pull-out data is from NAAMAN and SHAH (1976).

The composite crack opening $\delta$ may be divided into three contributions:

$$
\delta=\Delta_{1}+\Delta_{2}+\Delta_{3},
$$

where $\Delta_{1}=s_{1}(0)+s_{2}(0)$ is the slipping of the fiber ends, $\Delta_{2}$ is the (non-uniform) elastic stretching of the embedded length of the fiber, and $\Delta_{3}$ is the elastic stretching of the slipped out length bridging across the crack. Because of the frictional slip resistance, the fiber axial force varies along the embedded fiber length from zero at the fiber end to a maximum at the matrix crack where the fiber exits. If the fiber stress here reaches the fiber strength, then fiber rupture will occur and the slippage 


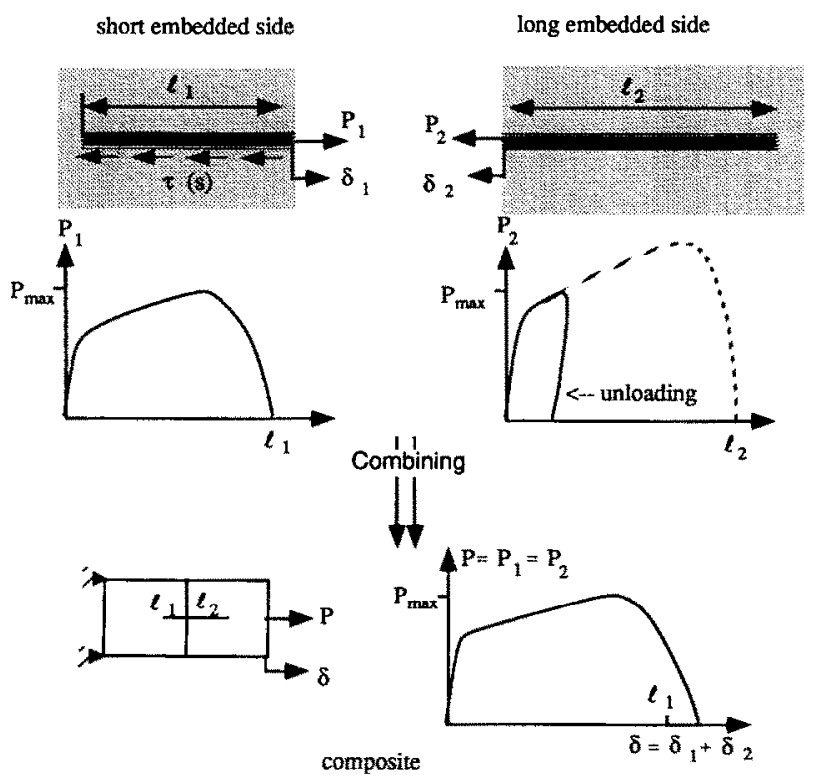

Fig. 4. Schematics of the modeling procedure by first envisioning the direct pull-out of each of the short and long embedded fiber segments and then combining them, based on compatibility and equilibrium considerations to calculate the $P-\delta$ curve.

process terminates. Naturally, that fiber will no longer contribute to the bridging stress in the composite. The fiber bridging force as a function of crack opening is illustrated schematically in Fig. 6, for fibers of different lengths at an inclining angle $\phi=0$.

For a fiber with the same embedded length $\ell$ and with the same end-slippage distance $(s(0))$, but with a non-zero inclining angle $\phi$ (Fig. 3b), the bridging force will be increased. This so-called "snubbing effect" has been demonstrated with a set of experiments of angle pull-out of synthetic fibers embedded in a cement matrix (LI et $a l$. , 1990). Figure 7 summarizes test results of normalized load as a function of inclining angle, for nylon and polypropylene fibers. These experimental data suggest that the snubbing effect may be modeled as if the fiber were pulled around a friction pulley, so that the bridging force for angle pull-out $\left(\left.P\right|_{\phi}\right)$ to that for $\phi=0\left(\left.P\right|_{\phi=0}\right)$ will be related by:

$$
\left.P\right|_{\phi}=\left.\mathrm{e}^{\mathrm{f} \phi} P\right|_{\phi=0},
$$

where the snubbing friction coefficient $f$ is an interface material parameter which must be determined experimentally for each fiber/matrix combination. The snubbing friction coefficients for nylon and polypropylene in normal strength mortar have been determined to be 0.99 and 0.70 respectively (LI et al., 1990). These values have been used to compute the two exponential curves shown in Fig. 7, which show reasonable agreement with the experimental data. However, for high angle pull-out $(\phi>60$ degrees), data scatter becomes significant due to sporadic matrix spalling. It should be noted that the distribution of the fiber axial force inside the matrix will still be the 
(a)

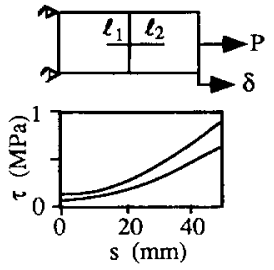

(b)

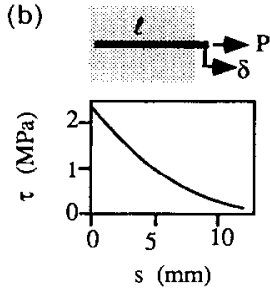

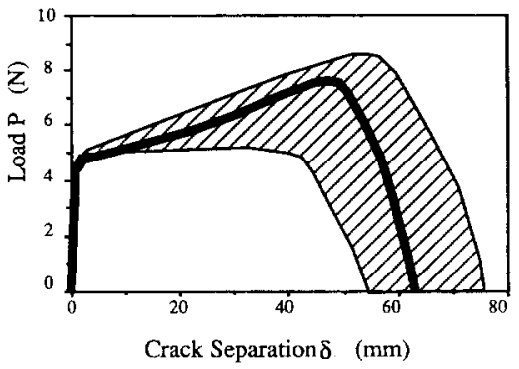

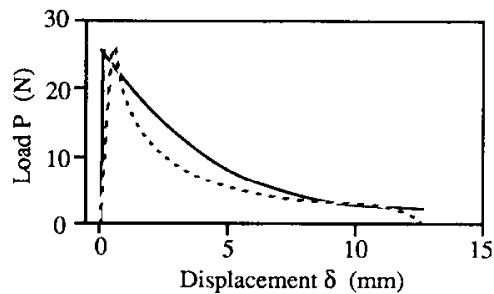

FIG. 5. Force-displacement relationship of single fiber pulled out from a cement matrix with test configurations shown. (a) Experimental data ( 4 sets) of nylon fibers fall in shaded area. Thick solid line is model prediction based on the slip-hardening curve shown in inset on left. (b) Experimental data for steel fiber is shown as dotted line. Solid line is model prediction based on the slip-hardening curve shown in insert on left.

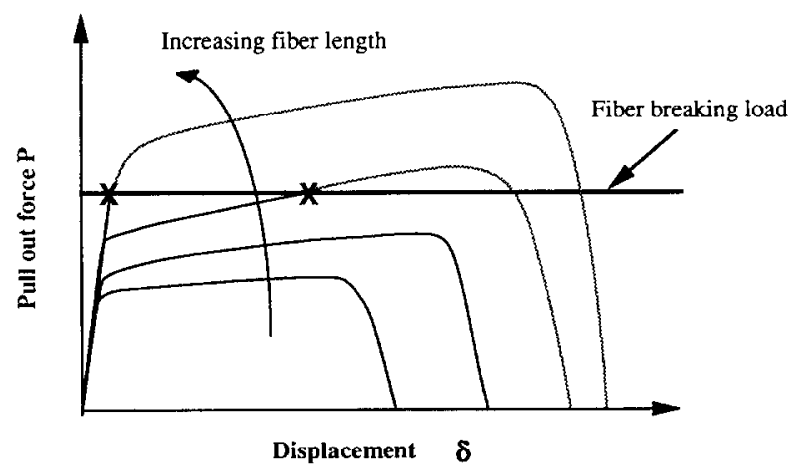

FIG. 6. Fiber bridging force shown schematically as a function of crack opening displacement for the problem defined in Fig. 3(a) $(\phi=0)$. Longer fibers contribute to a rising energy absorption. However, if the force rises too high, causing fiber stress to reach fiber strength, rupture of fiber occurs (Indicated by " $\mathrm{X}$ "), reducing the reinforcement effectiveness. 


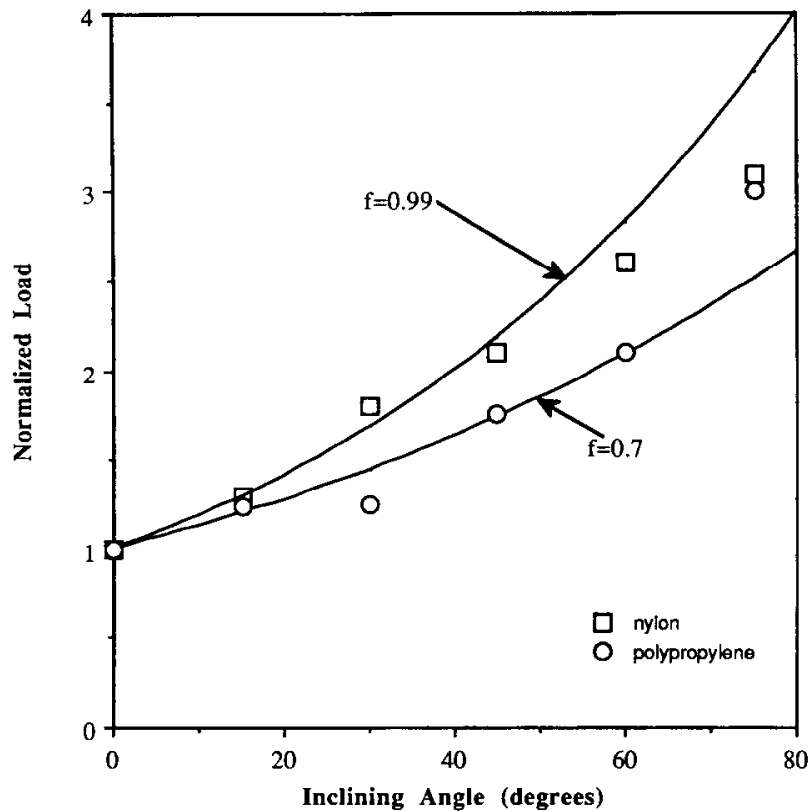

FIG. 7. Effect of inclining angle and snubbing friction on the fiber pull-out force. Averaged experimental data for nylon (square points) and polypropylene (circular points) are indicated. The normalized load is defined as the ratio of the maximum pull-out load to the embedded fiber length at arbitrary inclining angles $\phi$, normalized by the same ratio at $\phi=0$. The curves are simulations based on the friction pulley model.

See text for more details.

same for fibers of the same embedded length, irrespective of their inclining angles $\phi$ (Fig. 3b).

During the loading phase $(\mathrm{d} P / \mathrm{d} \delta \geqslant 0)$, the crack separation for inclined fibers can be calculated from that of $\phi=0$ (as per Eq. 7) using superposition to account for the snubbing-induced tensile stress increment in the bridging fiber segment:

$$
\left.\delta\right|_{\phi}=\frac{\left.P\right|_{\phi}-\left.P\right|_{\phi=0}}{E_{\mathrm{f}} A_{\mathrm{f}}} \Delta_{1}+\left.\delta\right|_{\phi=0},
$$

where $E_{\mathrm{f}}$ is the fiber elastic modulus. During unloading after reaching the peak load, part of the elastic elongation of the longer fiber embedded segment is recovered. The amount of the recovery depends on the snubbing friction force. Since the recovery occurs only after the shorter fiber segment has begun to slip out (i.e. when $s_{1}(0)>0$ ), and the magnitude of this recovery is, in general, much smaller than the slippage distance, the recovery during unloading is neglected for simplicity. By so doing, $\left.\delta\right|_{\phi}$ can always be determined from $\left.\delta\right|_{\phi=0}$ for the same $\ell$ from Eq. (9), without the need to calculate the pull-out response directly for each $\ell$ and $\phi$. Equations (8) and (9) provide the $P-\delta$ relation (for any $\phi$ ) sought for.

\section{Composite tension-softening curve $\sigma_{\mathrm{c}}(\delta)$}

To deduce the tension-softening curve for the composite, we may compute the traction transmitted across the matrix crack by integrating the force contributions 


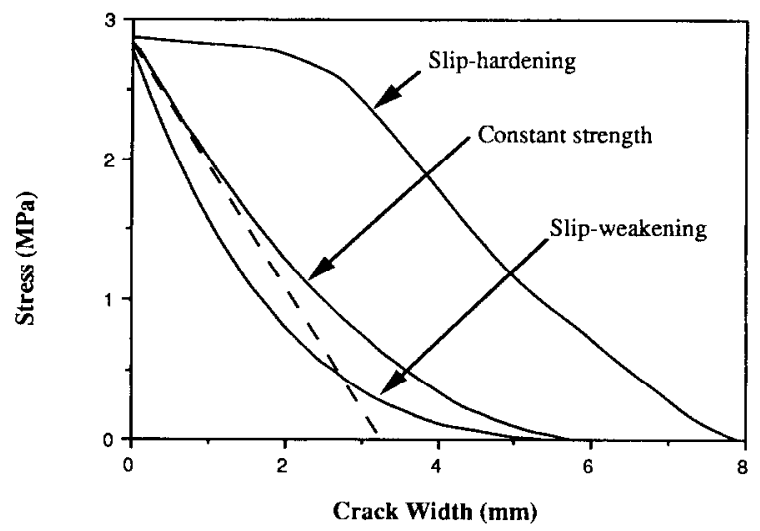

FIG. 8. Effect of interface bond type: model predicted tension-softening curves for composites with slip-hardening interfaces, represented by $\tau=1.02+0.2 s+0.2 s^{2}$; slip-weakening interfaces, represented by $\tau=1.02-0.2 s+0.01 s^{2}$; and constant strength interfaces, represented by $\tau=1.02(\tau$ in MPa, $s$ in $\mathrm{mm})$ The dashed line is also for the constant strength case but assuming an averaged embedded length of pullout instead of the present model formulation. See text for more details. Other parameters used in this calculation: $d_{\mathrm{f}}=38 \mu \mathrm{m}, L_{\mathrm{f}}=12.7 \mathrm{~mm}, V_{\mathrm{f}}=1 \%, E_{\mathrm{f}}=117 \mathrm{GPa}, f=0.7, \sigma_{u}^{\mathrm{f}}=2.6 \mathrm{GPa}$.

from those fibers which are active in the bridging action. Thus, for each crack opening $\delta$, the composite stress $\sigma_{\mathrm{c}}$ may be obtained from:

$$
\sigma_{\mathrm{c}}=\frac{F}{A_{\mathrm{c}}}=\frac{V_{\mathrm{f}}}{A_{\mathrm{f}}} \int_{z=0}^{L_{\mathrm{f}} / 2}\left[\int_{\phi=0}^{\arccos \left(2 z L_{\mathrm{i}}\right)} P(\ell, \phi, \delta) p(\phi) \mathrm{d} \phi\right] p(z) \mathrm{d} z .
$$

The constituent parameters needed for Eq. (10) are (1) fiber geometry - diameter $d_{\mathrm{f}}$ and length $L_{\mathrm{f}}$, (2) fiber properties-elastic modulus $E_{\mathrm{f}}$ and fiber strength $\sigma_{\mathrm{f}}^{u}$, (3) fiber volume fraction $V_{\mathrm{f}}$, and (4) properties of fiber-matrix interface-bond strength $\tau$ or slip-weakening/hardening law $\tau-s$, and snubbing friction coefficient $f$. The fracture energy of the composite may be obtained by integration of (10) with respect to the crack opening. Equation (10) suggests that the $\sigma_{\mathrm{c}}(\delta)$ scales with fiber volume fraction, and inversely with fiber diameter (since $P$ varies linearly with fiber diameter).

\section{Predicted 'Tension-Softening Curves}

The predicted tension-softening curves based on Eq. (10) are shown in Fig. 8, for the cases of a constant bond strength, slip-hardening and slip-weakening. The composite with a slip-hardening interface shows good retention of strength with a moderate amount of crack opening, prior to a more-or-less linear drop-off. In contrast, the composite with constant bond strength and that with slip-weakening show a rapid decay in strength with crack opening. This aspect of strength retention is important in considering composite post-cracking ductility. The critical opening when the tensile load-bearing capacity falls to zero is larger for the case of slip-hardening than for the 
other cases because some fibers can be pulled out from both sides. Thus, for composites with slip-hardening interfaces, the critical opening exceeds half the fiber length.

The tension-softening curve for a composite with fiber-matrix interface of constant bond strength may be expected to be approximately linear, indicated by the dashed line in Fig. 8, if it is assumed that all the fibers are pulled out with an expected embedment length of $L_{\mathrm{f}} / 4$ (e.g. Morton, 1979 ; Gopalaratnam and ShaH, 1987). In a randomly distributed fiber composite, the fibers are pulled out with varying amount of embedment length so that those with short embedment length will stop contributing at small crack openings and the composite load has to be assumed by the remaining bridging fibers. This results in the predicted nonlinear, decaying curve, as was first suggested qualitatively by ARGON et al. (1979). For the specific case shown in Fig. 8 , the fiber length is relatively short so that only about $3 \%$ of fibers are broken (see discussion to follow). For composites with longer fibers, more fibers are expected to be broken and the deviation of the tension-softening curve from a linear drop will be even more significant.

\section{Effect of Fiber Length on the Post-peak Tensile Properties}

For an aligned fiber-reinforced composite system, the optimal fiber length should be close to, but not more than, twice the critical fiber embedded length $L_{\mathrm{c}}$, defined as

$$
L_{\mathrm{c}}=\frac{d_{\mathrm{f}} \sigma_{\mathrm{f}}^{\prime \prime}}{4 \tau},
$$

so as to ensure that all fibers are pulled out with the maximum amount of frictional work and yet do not rupture. For the case of 3-D random distribution where the snubbing effect induces greater load on fibers pulled out at an angle, the optimal fiber length may be expected to be less than $2 L_{\mathrm{c}}$, depending on the magnitude of $f$. Figure 9 shows the calculated fracture energy by integrating $\sigma_{c}$ in (10) with respect to the crack opening $\delta$ and expressed as a function of the normalized fiber length for the two cases of $f=0.7$ and 1.0 , and for a fixed fiber volume fraction of $1 \%$. This calculation assumes a constant frictional bond strength $\tau$. The percentages of ruptured fibers corresponding to each fiber length are also shown. As expected, fibers arc completely pulled out when their lengths are short. As the fiber length approaches $L_{\mathrm{c}}$, the amount of ruptured fibers increases and eventually reaches $20-27 \%$ when the fiber length is twice $L_{c}$. Fiber rupture reduces the number of contributing fibers and their corresponding frictional work to the composite fracture energy. On the other hand, increased length enhances the bridging forces (see Fig. 6) and hence the composite fracture energy. These competing effects result in a maximum in the composite fracture energy $G$. For $f=0.7, G$ peaks at $L_{\mathrm{f}} \approx 1.4 L_{\mathrm{c}}$, whereas for $f=1.0, G$ peaks at $L_{\mathrm{f}} \approx 1.2 L_{\mathrm{c}}$.

Because of fiber rupture, the composite tension-softening behavior can be substantially altered. Figure 10 shows that a significant drop in load may occur for small crack opening in the case where the fiber length approaches or exceeds twice the critical length. This rapid load drop explains the reduction in $G$, even though the 

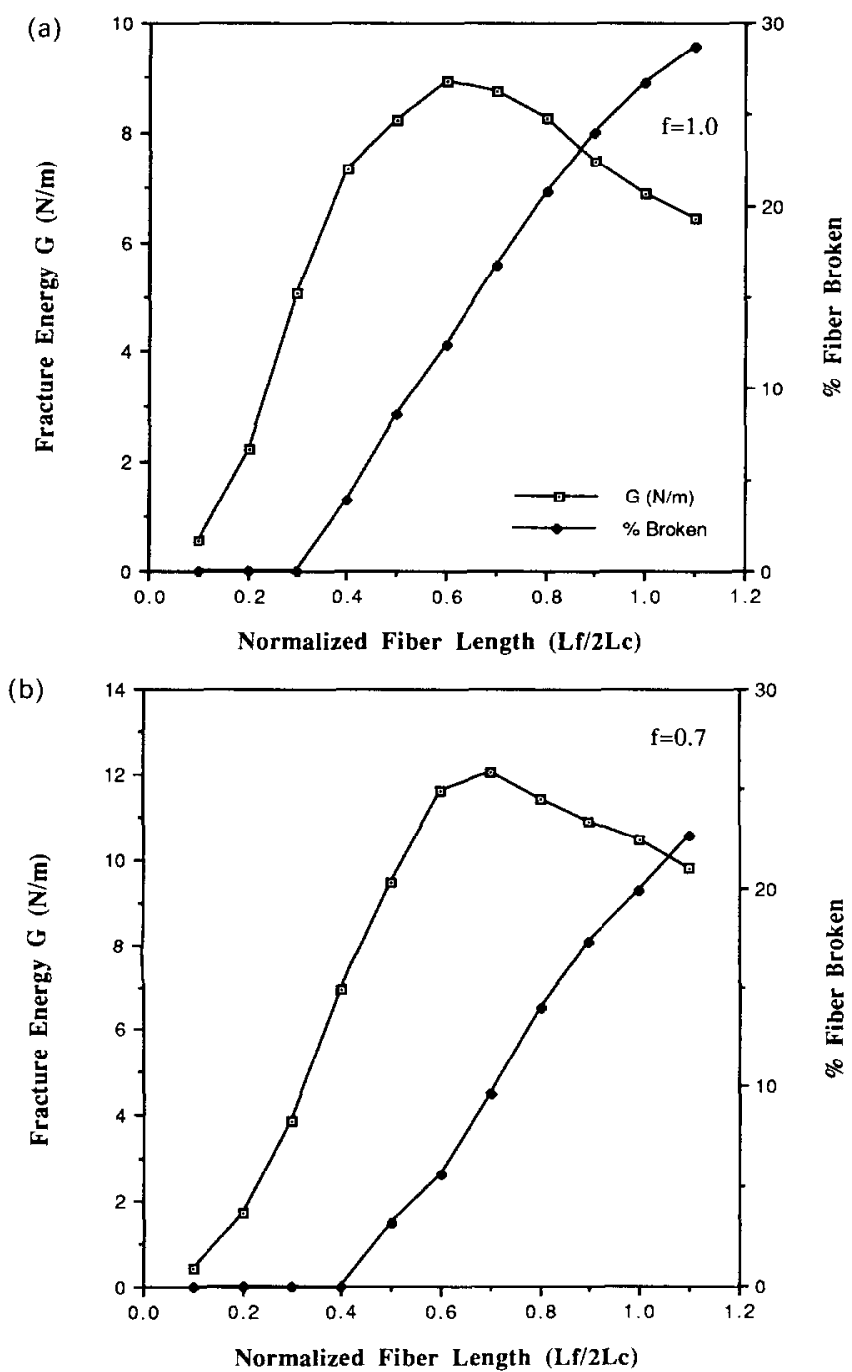

Fig. 9. Model-predicted fracture energy and percentage of fiber broken as a function of fiber length for (a) $f=1.0$ and (b) $f=0.7$. Other parameters used in this calculation: $d_{\mathrm{f}}=38 \mu \mathrm{m}, L_{\mathrm{f}}=12.7 \mathrm{~mm}, V_{\mathrm{f}}=1 \%$, $E_{\mathrm{f}}=117 \mathrm{GPa}, \sigma_{u}^{\mathrm{f}}=2.6 \mathrm{GPa}, \tau=2 \mathrm{MPa}$.

initial post-crack strength is higher than those composites with shorter fibers, despite the fact that complete fiber pull-out always occurs at larger crack opening when the fibers are longer.

We now turn our attention to a systematic study of the effect of fiber length on the fracture energy associated with fiber bridging. This effect was studied by COTTRELI. (1964) and COOPER and KeLly (1970), for the case of aligned, rigid and discontinuous fibers. They showed that when the fiber length is less than twice the critical embedded length, all fibers will be pulled out and the fracture energy will increase as the square 


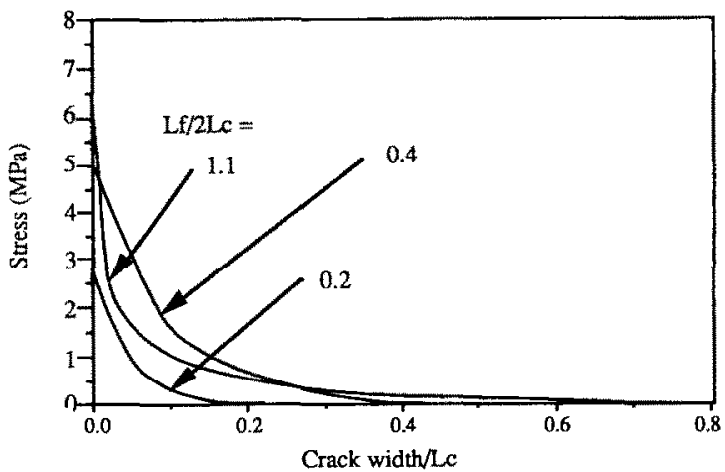

FIG. 10. Effect of fiber length: model-predicted tension-softening curves for composites with different fiber length but the same fiber volume fraction and diameter. This calculation is done for $d_{\mathrm{f}}=38 \mu \mathrm{m} ; V_{\mathfrak{f}}=1 \%$, $E_{\S}=117 \mathrm{GPa}, f=1.0, \sigma_{i}^{\mathrm{f}}=2.6 \mathrm{GPa}, \tau=2 \mathrm{MPa}$.

of the fiber length. However, when the fiber length exceeds twice the critical embedded length, some fibers will be ruptured and do not contribute to the work of fracture, such that the fracture energy will decrease as the inverse of the fiber length.

To facilitate the formulation of this problem in the case of random fibers which pull out with exit forces controlled by a snubbing friction, we have adopted in this section (specifically in relation to Fig. 11) the same assumption of fiber rigidity (in axial elongation but flexible in bending) and a constant (slip-independent) interfacial bond strength in the calculation of the composite bridging fracture energy. In this case, the energy absorption for a single fiber of embedded length $\ell$ pulled out at an angle $\phi$ can be shown to be equal to

$$
G_{s}=\frac{\pi}{2} d_{1} \tau \ell^{2} \mathrm{e}^{\mathrm{f} \phi} .
$$

Hence, analogous to the derivation of Eq. (10), the composite fracture energy could be computed from

$$
G=\frac{V_{\mathrm{f}}}{A_{\mathrm{f}}} \int_{z=0}^{L_{\mathrm{f}} / 2}\left[\int_{\phi=0}^{\arccos \left(2 z_{z} / L_{\mathrm{f}}\right)} G_{s}\left(\ell, f, d_{\mathrm{f}}, \phi\right) U\left(g\left(z, L_{\mathrm{f}}, L_{\mathrm{c}}, f\right)\right) p(\phi) \mathrm{d} \phi\right] p(z) \mathrm{d} z,
$$

where $U(g)$ is the step function such that

$$
U(g)=\left\{\begin{array}{ll}
1 & g>0 \\
0 & g \leqslant 0
\end{array},\right.
$$

$g$ is defined as follows:

$$
g=z-\left(\frac{L_{\mathrm{f}}}{2}-L_{\mathrm{c}} \mathrm{e}^{1 \mathrm{i} \phi}\right) \cos \phi
$$

and $p(z)$ and $p(\phi)$ are given in (3) and (4) for a uniformly random distribution of fiber location and orientation. The step function $U$ has been included in the integrand 
in (13) in order to discount those fibers which will be broken instead of pull-out (equivalent to stating that $g$ in (15) becomes negative). Equation (13) can be normalized to the following form:

$$
\tilde{G}=2 \tilde{L}_{f}^{2} F\left(\tilde{L}_{i}, f\right)
$$

where

$$
\begin{gathered}
\tilde{G} \equiv \frac{G}{\left(\tau V_{\uparrow} d_{f}\right)\left(L_{\mathrm{c}} / d_{\mathrm{f}}\right)^{2}}, \\
\tilde{L}_{\uparrow}=\frac{L_{\mathrm{f}} / 2}{L_{\mathrm{c}}}
\end{gathered}
$$

and the non-dimensional function $F$ is

$$
F=\int_{z^{\prime}=0}^{1} \int_{\phi=0}^{\cos { }^{\prime}\left(z^{\prime}\right)}\left[\begin{array}{c}
z^{\prime} \\
-\cos \phi
\end{array}\right]^{2} \mathrm{e}^{\mathrm{f} \phi} p(\phi) U\left[z^{\prime}-\left(1-\begin{array}{c}
1 \\
\tilde{L}_{i} \mathrm{e}^{\mathrm{f} \phi}
\end{array}\right) \cos \phi\right] \mathrm{d} \phi \mathrm{d} z^{\prime} .
$$

It can be shown that for aligned fiber systems, (16) reduces to (by setting $p(\phi)$ to be the Dirac delta function) the simple expressions

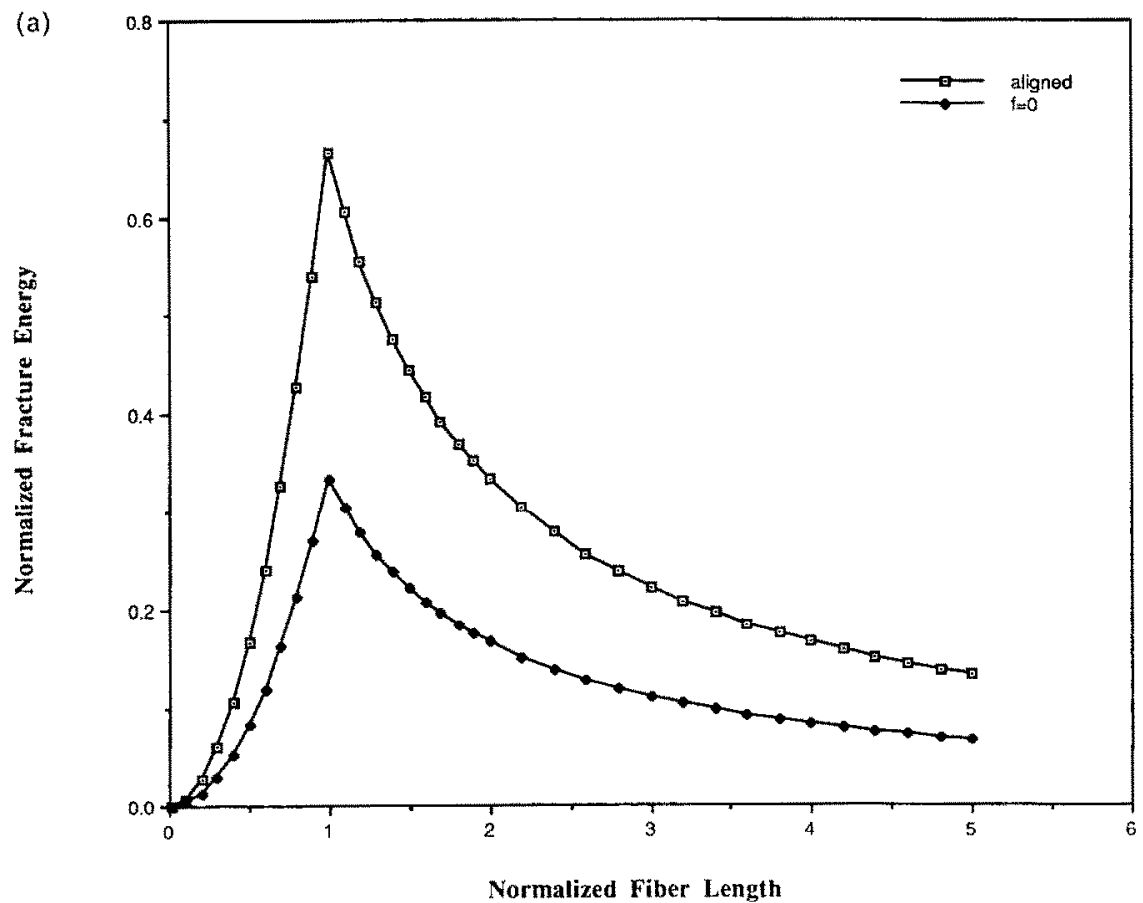

Fig. 11. Dependence of normalized fracture energy on normalized fiber length and snubbing friction. (a) Comparison between aligned case and random but no snubbing friction case. (b) Effect of snubbing friction coefficient on fracture energy. 


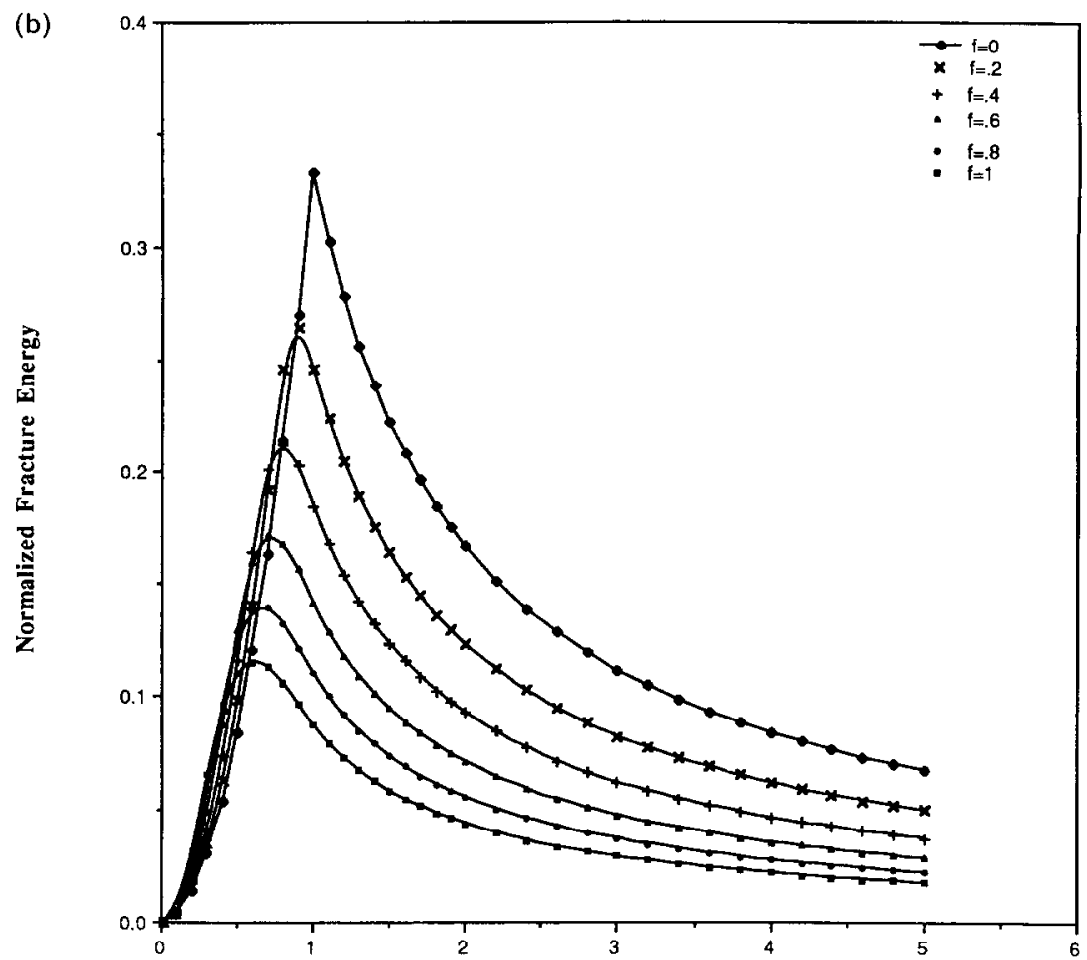

Normalized Fiber Length

FIG. 11. Continued.

$$
\tilde{G}=\frac{2}{3}\left\{\begin{array}{lll}
\tilde{L}_{f}^{2} & \text { for } & \tilde{L}_{\mathrm{f}} \leqslant 1 \\
1 / \tilde{L}_{\mathrm{f}} & \text { for } & \tilde{L}_{\mathrm{f}}>1
\end{array}\right.
$$

which were discussed by Cottrell (1964) and Cooper and Kelly (1970). Equation (16) generalizes the aligned cases to the random distribution case. The limiting case of no snubbing may be obtained by setting $f=0$, corresponding to a recent result of a study by WETHERHOLD (1989).

Figure 11 shows the normalized fracture energy $\tilde{G}$ as a function of $\tilde{L}_{\mathrm{f}}$, for a range of snubbing friction coefficients. The randomness of the fiber clearly reduces the fracture energy from that of the aligned case (Fig. 11a). However, for very short fibers $\left(L_{\mathrm{f}}<L_{\mathrm{c}}\right)$, the fracture energy is slightly improved by the snubbing effect. For longer fibers, the snubbing effect further reduces the fracture energy by causing a larger amount of fiber rupture, and causes the maximum fracture energy peaks to shif towards the shorter fiber lengths (Fig. 11b). Equations (16) and (17) reveal that for 
a given snubbing friction coefficient and fiber length, the fracture energy $G$ is directly proportional to $V_{\mathrm{f}}, \tau$, and $L_{\mathrm{c}}^{2}$ and inversely proportional to the fiber diameter. This shows the importance of ensuring large values of $L_{\mathrm{c}}$ for fracture energy optimization. In addition, comparison with the specific cases shown in Fig. 9 suggest that (16) gives $G$ within $10 \%$ error, despite the simplifying assumptions of fiber rigidity and the ignoring of the pre-peak (frictional debonding) energy absorption in the fiber pullout process in arriving at (12) and (16).

\section{Comparisons of Model Predictions with Experimental Observations}

The model parameters used for predicting the tension-softening curve of two mixes of Spectra 900 fiber-reinforced mortar are given in Table 1. Both the model-predicted curve and the experimentally determined curve based on specially devised uniaxial testing technique (WANG et al., 1990a,b) are presented in Fig. 12. In Mix S1 the matrix material is a normal strength mortar with tensile strength of approximately $2.5 \mathrm{MPa}$. From SEM studies of the fracture surface, a large amount of surface spall was observed, while the Spectra fibers do not appear to be abraded (Wang et al.,

TABLE 1. Material and geometry parametric values used in the prediction of the tension-softening curves in Fig. 12

\begin{tabular}{lcc}
\hline Parameters & Mix S1 & Mix SH \\
\hline$d_{\mathrm{r}}(\mu \mathrm{m})$ & 38 & 38 \\
$L_{\mathrm{f}}(\mathrm{mm})$ & 12.7 & 6.3 \\
$E_{\mathrm{f}}(\mathrm{GPa})$ & 120 & 120 \\
$\sigma_{\mathrm{f}}(\mathrm{GPa})$ & 2.6 & 2.6 \\
$V_{\mathrm{f}}(\%)$ & 1 & 0.6 \\
$\tau(\mathrm{MPa})$ & 1.02 & 1.02 \\
$f$ & 0.7 & 0.7 \\
\hline
\end{tabular}

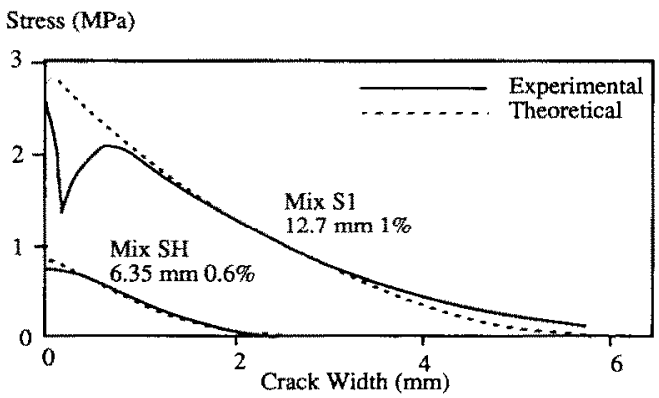

Fic. 12. Comparisons of model-predicted tension-softening curves for two mixes of Spectra fiber-reinforced mortar. Mix SI uses a normal strength matrix. Mix SH uses a high strength matrix. Other parameters can be found in Table 1 . 
1990c). In contrast, GREEN (1989) observed no spalling in the high strength mortar matrix Mix SH with a tensile strength of approximately 3.4 MPa. Although abrasion of Spectra 900 fibers has been observed under the SEM (GREEN, 1989) in a similar mix, we have used the same constant bond strength as for Mix S1 on account of the short length of the fiber used in the Mix SH. The experimental testing technique and data on bond strength of various synthetic fibers can be found in WANG et al. (1987, 1988a) and Li et al. (1990). For the snubbing friction coefficient $f$, angle pull-out data for the olefin (polypropylene) from a cement matrix was used, although a direct measurement of $f$ of Spectra fiber embedded in both types of mortar matrix would have been preferred.

The comparisons shown in Fig. 12 suggest that the model predictions are reasonable, with the exception of the initial part of the curve for Mix S1, which may reflect matrix spalling effects not accounted for in the model. The bridging fracture energy, which is simply the area under the tension-softening curves, also appears to be well predicted. The agreement between experimental results and theoretical predictions, however, should be interpreted with caution, due to model assumptions mentioned earlier and the use of a snubbing coefficient measured from a pull-out test of polypropylene rather than polyethylene.

\section{FurTHER Discussion}

It is a general notion that an aligned fiber composite system loaded in the direction of alignment is more efficient in terms of post-cracking tensile load capacity than a random fiber composite system, because of an expected larger amount of fiber crossing the matrix crack. However, it appears possible that this loss of reinforcement efficiency in a randomly distributed fiber composite could be partially made up by means of the snubbing effect described in this paper. In terms of composite fracture energy due to fiber bridging, the snubbing effect contributes positively only in the case of very short fibers (typically $L_{\mathrm{f}}<L_{\mathrm{c}}$ ). This implies that increasing $L_{\mathrm{c}}$ in random fiber composites (for all $f$ ) becomes even more important than for aligned fiber composites for which maximum fracture energy can be achieved by ensuring $L_{\mathrm{f}} \approx 2 L_{\mathrm{c}}$.

The snubbing effect in synthetic fiber-reinforced mortar composites is analogous to the plastic bending effect in steel fiber-reinforced mortar in which fibers must bend and deform plastically as they exit at an angle to the matrix crack. However, there is a distinctive difference between this bending effect and the snubbing effect regarding their contribution to the composite strength $a$.nd fracture energy. Because plastic bending can be considered a property of the fiber yield strength only and is independent of the interface strength, this bending effect contributes additively to composite strength and fracture energy in addition to the interface frictional effect. In contrast, the snubbing friction effect depends directly on the interface property and must contribute multiplicatively to the composite properties, as implied by Eqs (8) and (10). This difference is particularly interesting in light of the fiber abrasion contribution to the interface slip-hardening phenomenon found in some synthetic fibers/mortar matrix systems (WANG el al., 1988b). These aspects have yet to be fully exploited in composite design. 


\section{CONCLUSIONS}

This paper presents a theoretical model of post-peak behavior of short fiberreinforced brittle matrix composites with particular attention to the tension-softening behavior of synthetic fiber-reinforced mortars, for which comparisons were made between experimental data and theoretical predictions. The model accounts for several physical processes related to the material structure and which appear to govern the tensile properties in such composites. These physical processes include the effect of fiber abrasion during fiber pull-out and result in a slip-hardening interfacial bond strength, as well as a snubbing effect exhibited during fiber withdrawal inclined to the matrix crack. The randomness of the fiber location and orientation is accounted for statistically.

It is found that the composite post-crack tension-softening curve and fracture energy are strongly influenced by the snubbing effect of inclined fiber pull-out. The composite fracture energy first increases with fiber length then decreases due to fiber rupture. The maximum fracture energy (peaks in Fig. $11 \mathrm{~b}$ ) tends to shift from $L_{f}=2 L_{4}$ to $L_{\mathrm{f}}=L_{\mathrm{c}}$ as $f$ increases from 0 to 1 . The amount of fiber rupture depends on the bond strength, fiber length and on the snubbing coefficient. While predictions made by the present composite model are encouraging, this model must be applied with care for material systems for which the model assumptions are grossly violated such as in the case where fiber dispersion is inadequate. However, the systematic formulation of the problem should allow flexibility in relaxing certain restrictive assumptions so that the model may be expected to have broader implications other than those discussed in this paper.

\section{ACKNOWLEDGEMFNTS}

The authors thank two anonymous reviewers for their helpful comments which led to several improvements over the original version of this paper and J. HUANG who provided numerical computational assistance leading to Fig. 11. We gratefully acknowledge financial support of this work from the National Science Foundation, the Shimizu Corporation, E.I. du Pont Nemours \& Co. Inc. and (for V.C.L.) the University of Michigan.

\section{REFERENCES}

Akgun, A. S., Hawkins, G. W. and Kuo, H. Y.

Aveston, J., CoOper, G. A. and KELLY, A.

BUDIANSKY, B., HutCHINSON, J. W. and Evans. A. G.

Cooper, G. A. and Kelly, A.
1979

1971

1986

1970
J. Mater. Sci. 14, 1707.

In The Properties of Fiber Composites, p. 15. Conference Proceedings, National Physical Laboratory, IPC Science and Technology Press Ltd, London.

J. Mech. Phys. Solids 34, 167.

In Mechanics of Composite Materials (edited by $F$. W. WENDT, $H$. LIEBowITZ and $N$. Perrone), p. 653. Pergamon Press, Oxford. 
Cottrell, A. H.

GaO, Y. C., MaI, Y. W. and CotTrell, $B$.

Gopalaratnam, V. S. and Shah, S. P.

GreEN, E.

Hillerborg, A.

Kelly, A. and Macmillan, N. H. 1986

LI, V. C. and LIANG, E.

LI, V. C. and WARD, R. J.

LI, V. C., WANG, Y. and

BACKER, $\mathrm{S}$.

MORTON, J.

NaAman, A. and Shah, S. P.

RICE, J. R.

WANG, Y., BACKeR, S. and LI, V. C.

WANG, Y., LI, V. C. and BACKER, $S$.

WANG, Y., LI, V. C. and BACKER, $S$.

WANG, Y., LI, V. C. and BACKER, $S$.

WANG, Y., LI, V. C. and BACKER, $S$.

WANG, Y., LI, V. C. and BACKER, $S$.

WARD, R. and LI, V. C.

Wetherhold, R. C.
1964 Proc. R. Soc. A282, 2.

1988 J. Appl. Math. Phys. (ZAMP) 39, 550.

1987 J. Engng Mech. ASCE 113, 635.

1989 M.S. Thesis, MIT, Cambridge, MA.

1989 In Applications of Fracture Mechanics to Concrete (edited by V. C. Li and Z. BazanT). American Concrete Institute Special Publication.

Strong Solids. Oxford Science Publications, Oxford University Press, New York.

1986 J. Engng Mech. ASCE 112, 566.

1990 To appear in Proc. RILEM International Workshop on Applications of Fracture Mechanics to Reinforced Concrete, Torino, Italy.

1990 J. Compos. 21, 2, 132.

1979 Materiaux et Constructions 12, 393.

1976 J. Struct. ASCE 102, 1537.

1968 In Fracture: An Advanced Treatise 2, 191. Academic Press Inc., New York.

1987 J. Mater. Sci. 22, 4281.

1988a Mater. Res. Soc. Symp. Proc. 114, 159.

1988b Int. J. Cement Compos. Lightweight Concr. 10, 143.

1990a Am. Concr. Inst. Mater. J. 87, 461.

1990 b J. Cement Concr. Compos. 12, 29.

1990c Accepted for publication in J. Mater. Sci.

1990 Accepted for publication Am. Concr. Inst. Struct. $J$.

1989 ASME Trans. 89-GT-125. 